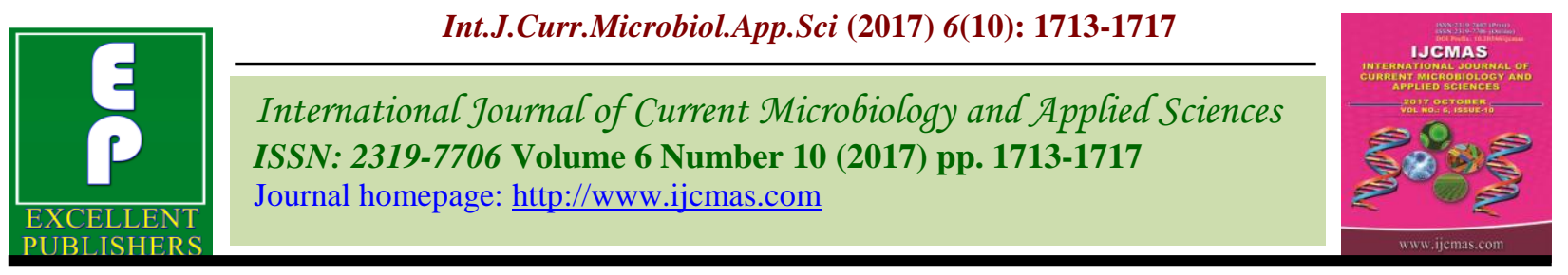

Original Research Article

https://doi.org/10.20546/ijcmas.2017.610.207

\title{
Seroprevalence of Chikungunya among Febrile Patients in a Tertiary Care Hospital
}

\author{
Mita D. Wadekar, J.V. Sathish* and Trupti B. Naik \\ Department of Microbiology, Chamarajanagar Institute of Medical Sciences, \\ Chamarajanagar, Karnataka, India \\ *Corresponding author
}

\section{A B S T R A C T}

Keywords

Chikungunya, Seroprevalence, ELISA.

Article Info

Accepted:

17 September 2017

Available Online:

10 October 2017
Chikungunya fever is a self-limiting viral fever caused by an arbovirus transmitted to human through mosquitoes. The sudden onset of very high fever along with rash and severe arthralgia are main symptoms. It still persists as major public health problem. Aim of the study is to know the seroprevalence of chikungunya infection in a tertiary-care hospital in Chamarajanagar, Karnataka, India. Data was collected from Microbiology Laboratory registers retrospectively for a period of one year from June 2016 to May 2017. Blood samples were collected from suspected cases of chikungunya fever and tested for IgM antibody using ELISA. Analysis was done using MS Excel. A total of 1432 chikungunya suspected serum samples were tested, of which $117(8.17 \%)$ samples were positive for IgM antibody. Maximum number of positive cases was in age group of 18-45 years $71(60.68 \%)$ with no significant difference between males $64(54.70 \%)$ and females $53(45.30 \%)$. Seroprevalence of chikungunya in our study was $8.17 \%$ which suggests that it still exists in our setup and indicates the need of appropriate strategies to reduce the severity of disease.

\section{Introduction}

Chikungunya fever is a tropical arboviral disease caused by chikungunya virus (CHIKV) that belongs to the family Togaviridae, genus Alphavirus (Tatjana Vilibic-Cavlek et al., 2015). The meaning of Chikungunya (CHIK) is "that which bends up" describing the stooped posture due to arthritic feature of the disease (Divya et al., 2016). It is transmitted to humans by Aedes aegypti and Aedes albopictus (Alessandra Lo Presti et al., 2014). During epidemics, humans are the primary reservoirs. Monkeys, rodents, birds, and cattle have been identified as animal reservoirs (Norbert G. Schwarz et al., 2012). Vertical transmission of disease from mother to child has also been documented (Meghna R. Sebastian et al., 2009).

Clinical manifestations are very variable, from asymptomatic illness to severe debilitating disease. Children are among the group at maximum risk for severe manifestations of the disease and some clinical features in this group are distinct from those seen in adults (Meghna R. Sebastian et al., 2009). Common signs and symptoms of an acute infection include polyarthralgia, high 
fever, asthenia, headache, vomiting, rash and myalgia. The Chikungunya infection often leads to prolonged joint pain, contributing to the long-lasting disease burden (Sanyaolu et al., 2016). The disease is rarely fatal but is associated with significant morbidity.

The illness has an approximate incubation period of 1-2 weeks (Kibet Sergon et al., 2008). The acute phase lasts for 2- 3 days and may remit for 1- 2 days after a gap of 4- 10 days resulting in a "saddle back" fever curve (Mohanty et al., 2013). Most infections completely resolve within weeks but there are reported cases of $\mathrm{CHIK} \mathrm{V}$ induced arthralgia lasting for months or even for years in the form of recurrent or persistent episodes (Narayan Shrihari et al., 2012).

The virus was first isolated in blood samples obtained during an epidemic of a "denguelike" disease that occurred between 1952 and 1953 in Tanzania (Cunha et al., 2017). CHIKV is an emerging arbovirus that is widespread in tropical regions and is spreading rapidly to temperate climates with recent epidemics in Africa and Asia and also documented outbreaks in Europe and America (Akinola et al., 2017). It was unknown to Indian population till it appeared as a major epidemic in 2006. Around 1.38 million Indian populations were affected by this disease. The states that first affected were Andhra Pradesh, Karnataka, Maharashtra, Madhya Pradesh, Tamil Nadu, Gujarat, and Kerala. All ages and both sexes were affected (Sakhiya et al., 2015). Resurgence of chikungunya has been attributed to various factors including globalization, increase in the mosquito population, loss of herd immunity and the mutation $\mathrm{A} 226 \mathrm{~V}$ in the $\mathrm{E} 1$ gene causing a significant increase in CHIKV infectivity for Ae. Albopictus (Cecilia, 2014).

In India, National Institute of Virology (NIV), Pune, is a WHO collaborating center for arboviral diseases. It is engaged in diagnosis, outbreak investigations and preparations of reagents for diagnosis of arboviral infections (Mohanty et al., 2013). Infections with CHIKV are confirmed by the detection of the virus, viral RNA, or CHIKV-specific antibodies in patient samples. Enzyme-linked immunosorbent assays (ELISAs) detect both anti-CHIKV immunoglobulin (Ig) $\mathrm{M}$ and IgG antibodies from either acute- or convalescentphase samples.

Testing of samples from imported cases found that CHIKV-specific IgM antibodies develop rapidly (typically present by 5-7 days after illness onset but noted as early as the day of illness onset) and persist for several months (Erin Staples et al., 2009). Chikungunya virus mutation, adaptation to new vectors, and spread to temperate regions signifies the seriousness of CHIKV as a global threat (Divya et al., 2016). Hence the study was undertaken to know the seroprevalence of chikungunya virus infection.

\section{Materials and Methods}

A retrospective study was conducted at Chamarajanagar Institute of Medical Sciences, Chamarajanagar from June 2016 to May 2017.

The age, gender and clinical details of patients and results of CHIK kit tests were collected from the laboratory registers. The data were entered into Excel for analysis. Permission was obtained from the institution. As the study was based on secondary data, there were no ethical issues.

Blood samples were collected from suspected cases of chikungunya. The serum was separated by centrifugation of the whole blood sample and tested for CHIK IgM antibody using IgM antibody capture ELISA kit produced by NIV (Arbovirus Diagnostic NIV, Pune, India). The tests were carried out following the manufacturer instruction. 


\section{Results and Discussion}

A total of 1432 chikungunya suspected serum samples were tested, of which $117(8.17 \%)$ samples were positive. Although all ages are affected, maximum number of positive cases was in age group of 18-45 years $71(60.68 \%)$ followed by age groups $1-18$ years $29(24.79 \%)$ and $>45$ years $17(14.53 \%)$. There was no significant difference between males $64(54.70 \%)$ and females 53(45.30\%) (Tables 1 and 2).

CHIKV is a flaviviral infection transmitted to humans through the bite of an infected female mosquito of the genus Aedes. Since, its first identification in the year 1952-53, both sporadic cases and major epidemics of CHIKV infection have been reported (Anuja P. Kawle et al., 2017). In India CHIKV outbreak was first reported in 1963 in Kolkata which accounted nearly 200 Deaths (Sai Gopal DVR et al., 2013). Major outbreaks that increased concerns about CHIKV occurred in India in 2005, where it affected 1.4 million people. More than one million cases of CHIKV infection were reported from different regions of India during January 2006 to August 2007, with most patients coming from Kerala and Tamil Nadu (Braira Wahida et al., 2017).

Table.1 Seroprevalence of chikungunya cases

\begin{tabular}{|c|c|}
\hline Total no. of samples tested & No. of positive samples NO. (\%) \\
\hline 1432 & $117(8.17 \%)$ \\
\hline
\end{tabular}

Table.2 Age and gender wise distribution of chikungunya cases

\begin{tabular}{|c|c|c|c|}
\hline \multirow{2}{*}{$\begin{array}{c}\text { Age } \\
\text { (years) }\end{array}$} & $\begin{array}{c}\text { No. of positive } \\
\text { samples } \\
\text { NO. }(\%)\end{array}$ & \multicolumn{2}{|c|}{ Gender } \\
\cline { 3 - 4 } & MO. $(\%)$ & F \\
\hline $\mathbf{1 - 1 8}$ & $29(24.79)$ & $10(08.55)$ & $19(16.24)$ \\
\hline $\mathbf{1 8 - 4 5}$ & $71(60.68)$ & $43(36.75)$ & $28(23.93)$ \\
\hline$>\mathbf{4 5}$ & $17(14.53)$ & $11(09.40)$ & $06(05.13)$ \\
\hline Total & $117(100)$ & $64(54.70)$ & $53(45.30)$ \\
\hline
\end{tabular}

Chikungunya is associated with joint pains lasting up to six months and the morbidity and disability caused due to chikungunya are enormous (Dikid et al., 2013). Typically, joint damage fluctuates over time, but always affects the same parts of the body, mostly the extremities (hands, ankles, knuckles).

The mortality rate is low $(0.4 \%)$, but is higher in babies less than 1 year old $(2.8 \%)$ and increases in the elderly with concurrent diseases (Girish Shakuntal et al., 2016). In some patients, Chikungunya can progress to cause chronic arthritis. The risk factors identified in the literature for persistent joint pains are age over 60 years, high viral load during acute illness, those with C- Reactive Proteins (CRPs) closer to upper limit of normal, absence of thrombocytopenia and only mild elevation of transaminases were more likely to develop persistent arthralgia (Vikram Londhey et al., 2016).

In this study, there was no significant difference between males $54.70 \%$ and females $45.30 \%$. In some studies, men 
appeared to be more susceptible to dengue infection whereas in others, women were more affected, and no gender differences were noted in others. This inconsistency may relate to gender differences in exposure to infection due to community-specific habits, customs or behaviours (Sissoko et al., 2008 and Azami et al., 2013).

This study showed seroprevalence of $8.17 \%$ with maximum number of positive cases in the age group of $18-45$ years $60.68 \%$. One of the study revealed differences in awareness of chikungunya, cause of the disease, vector responsible, mode of transmission, biting time and elimination of breeding of mosquitoes statistical significant among high and low incidence wards of all the States (Nagpal et $a l ., 2012$ ). The geographical distribution had a significant influence on the prevalence of antibodies to the virus. This might be explained by the possible impact of ecological characteristics of the areas on the natural cycles of the arthropod-borne viruses under consideration (Narayan Shrihari et al., 2012). Chikungunya fever is self-limiting disease and treatment is mainly supportive (Arunasree et al., 2015).

Infection with CHIKV is an emerging public health threat that should be addressed through prevention. Because there is no vaccine against $\mathrm{CHIKV}$, the cornerstone of prevention is to reduce Ae. aegypti mosquito populations by vector control strategies, mass education of the community by medical and paramedical staff at primary health centers, clearing stored water (mainly in construction sites) and personal protective measures (Kibet sergon et al., 2007 and B. Arunasree et al., 2015).

Chikungunya fever is self-limiting disease. Its existence in our setup indicates efforts have to be made through community awareness, vector control measures and early institution of supportive therapy.

\section{References}

Akinola, M. T., EL-Yuguda, A. D., Bukbuk, D. N. and Baba, S. S. 2017. Prevalence of IgG and $\operatorname{IgM}$ antibodies to Chikungunya virus among outpatients with febrile illness attending University of Maiduguri Teaching Hospital, Maiduguri, Borno State, Nigeria. African Journal of Microbiology Research, 11(7): 306-311.

Alessandra Lo Presti, Alessia Lai, Eleonora Cella, Gianguglielmo Zehender, Massimo Ciccozzi. 2014. Chikungunya virus, epidemiology, clinics and phylogenesis: A review. Asian Pacific Journal of Tropical Medicine, 925-932.

Anuja P. Kawle, Amit R. Nayak, Shradha S. Bhullar et al., 2017. Seroprevalence and clinical manifestations of chikungunya virus infection in rural areas of Chandrapur, Maharashtra, India. J Vector Borne Dis, 54: 35-43.

Arunasree, B., Prasad Uma, B. Rajsekhar. 2015. Epidemiology of Chikungunya Fever in Srikakulam District. Journal of Evidence based Medicine and Healthcare, 2(19): 2931-2935.

Azami, et al., 2013. Emergence of chikungunya seropositivity in healthy Malaysian adults residing in outbreak-free locations: Chikungunya seroprevalence results from the Malaysian Cohort. BMC Infectious Diseases, 13:67.

Braira Wahida, Amjad Alia, Shazia Rafiquea, Muhammad Idreesa, 2017. Global expansion of chikungunya virus: mapping the 64-year history. International Journal of Infectious Diseases, 58: 69-76.

Cecilia, D., 2014. Current status of dengue and chikungunya in India. WHO South-East Asia J Public Health, 3(1): 22-27.

Cunha, R.V., Trinta KS, Montalbano CA, Sucupira MVF, de Lima MM, Marques E, et al., 2017. Seroprevalence of Chikungunya Virus in a Rural Community in Brazil. PLoS Negl Trop Dis, 11(1): 1-11.

Dikid, T., S.K. Jain, A. Sharma, A. Kumar and J.P. Narain. 2013. Emerging \& re-emerging infections in India: An overview. Indian J Med Res, 138:19-31. 
Divya, P., Krishna S. 2016. Seroprevalence of Chikungunya virus infection in Ballari and nearby districts of Karnataka. International Journal of Medical Microbiology and Tropical Diseases, 2(4):175-17.

Erin Staples, J., Robert F. Breiman, and Ann M. Powers. 2009. Chikungunya Fever: An Epidemiological Review of a Re-Emerging Infectious Disease. Clinical Infectious Diseases, 49: 942-8.

Girish Shakuntal, Prasanna Parashare. 2016. A Study of Prevalence of Chikungunya among Children: A Cross-Sectional Study. Sch. J. App. Med. Sci., 4(5F):1857-1859.

Kibet sergon, et al., 2007. Seroprevalence of chikungunya virus infection on Grande Comore Island, Union of the Comoros, 2005. Am. J. Trop. Med. Hyg., 76(6): 11891193.

Kibet Sergon, et al., 2008. Seroprevalence of Chikungunya Virus (CHIKV) Infection on Lamu Island, Kenya, October 2004. Am. J. Trop. Med. Hyg., 78(2): 333-337.

Meghna R. Sebastian, Rakesh Lodha and S.K.Kabra. 2009. Chikungunya Infection in Children. Indian Journal of Pediatrics, 76:185-189.

Mohanty, I., Dash M, Sahu S, Narasimham MV, Panda P, Padhi S. 2013. Seroprevalence of chikungunya in southern Odisha. J Fam Med Primary Care, 2: 33-36.

Nagpal B.N., et al., 2012. Retrospective study of chikungunya outbreak in urban areas of India. Indian J Med Res, 135: 351-358.

Narayan Shrihari, Kumudini T. S, Mariraj. J, Krishna. S. 2012. The prevalence of chikungunya arboviral infection in and around Bellary district, Karnataka. Journal of Evolution of Medical and Dental Sciences, 1(5): 677-681.
Norbert, G., Schwarz et al., 2012. Seroprevalence of Antibodies against Chikungunya, Dengue, and Rift Valley Fever Viruses after Febrile Illness Outbreak, Madagascar. Emerging Infectious Diseases, 18(11): 1780-1786.

Sai Gopal, D.V.R., Naresh Kumar CVM. 2013. Isolation and diagnosis of Chikungunya virus causing outbreaks in Andhra Pradesh, India. J Clin Sci Res, 2:2-7.

Sakhiya, A.J., Gamit M, Prajapati K, Patel D, Shah P. 2015. Seroprevalence of Chikungunya cases in a tertiary-care hospital in Ahmedabad. Int $J$ Med Sci Public Health, 4:1297-1300.

Sanyaolu, A., Okorie C, Badaru O, Wynveen E, White S, Wallace W, et al., 2016. Chikungunya Epidemiology: A Global Perspective. SM J Public Health Epidemiol, 2(2): 1028.

Sissoko, D., Moendandze A, Malvy D, Giry C, Ezzedine K, et al., 2008. Seroprevalence and Risk Factors of Chikungunya Virus Infection in Mayotte, Indian Ocean, 20052006: A Population-Based Survey. PLoS ONE, 3(8).

Tatjana Vilibic-Cavlek et al., 2015. Seroprevalence and Entomological study on Chikungunya virus at the Croatian Littoral. Acta Microbiologica et Immunologica Hungarica, 62 (2): 199-206.

Vikram Londhey, Sachee Agrawal, Nilima Vaidya, Seema Kini, JS Shastri, Sujatha Sunil. 2016. Dengue and Chikungunya Virus Co-infections: The Inside Story. Journal of the Association of Physicians of India, 64: 36-40.

\section{How to cite this article:}

Mita D. Wadekar, J.V. Sathish and Trupti B. Naik. 2017. Seroprevalence of Chikungunya among Febrile Patients in a Tertiary Care Hospital. Int.J.Curr.Microbiol.App.Sci. 6(10): 17131717. doi: https://doi.org/10.20546/ijcmas.2017.610.207 